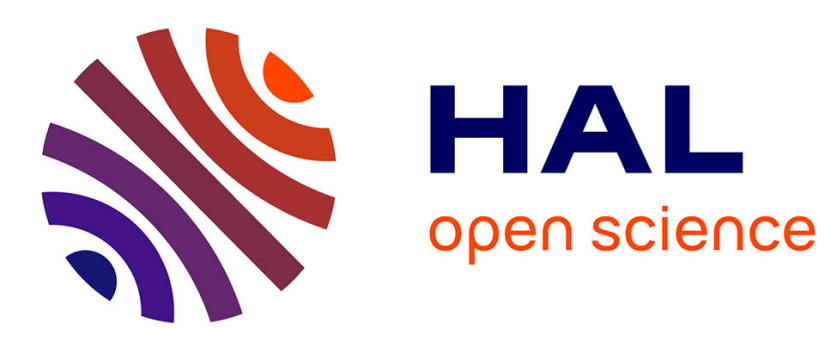

\title{
Le Canoë dans le potlatch lekwiltoq
}

\author{
Marie Mauzé
}

\section{To cite this version:}

Marie Mauzé. Le Canoë dans le potlatch lekwiltoq. L'Homme - Revue française d'anthropologie, 1989, 29 (109), pp.117-128. 10.3406/hom.1989.369086 . halshs-01324573

\section{HAL Id: halshs-01324573 https://shs.hal.science/halshs-01324573}

Submitted on 6 Jun 2016

HAL is a multi-disciplinary open access archive for the deposit and dissemination of scientific research documents, whether they are published or not. The documents may come from teaching and research institutions in France or abroad, or from public or private research centers.
L'archive ouverte pluridisciplinaire HAL, est destinée au dépôt et à la diffusion de documents scientifiques de niveau recherche, publiés ou non, émanant des établissements d'enseignement et de recherche français ou étrangers, des laboratoires publics ou privés. 


\section{Le Canoë dans le potlatch lekwiltoq}

Marie Mauzé

\section{Citer ce document / Cite this document :}

Mauzé Marie. Le Canoë dans le potlatch lekwiltoq. In: L'Homme, 1989, tome 29 n¹09. pp. 117-128;

doi : 10.3406/hom.1989.369086

http://www.persee.fr/doc/hom_0439-4216_1989_num_29_109_369086

Document généré le 29/03/2016 


\section{Le Canoë dans le potlatch lekwiltoq}

Marie MauzÉ, Le Canoë dans le potlatch lekwiltoq. - Les Lekwiltoq, l'une des principales tribus des Kwagul méridionaux, assignent au canoë dans leurs potlatch la place qu'occupent les Cuivres dans les autres groupes. Si un mythe semble justifier l'absence de Cuivres dans le potlatch lekwiltoq, l'ethnographie ne permet pas de poser une équivalence entre canoë et Cuivre, même si l'importance du canoë dans la vie sociale et cérémonielle de cette société guerrière est bien attestée. Pratiquement disparu chez les autres Kwagul, le potlatch à canoë s'est au contraire maintenu chez les Lekwiltoq pour devenir, à la fin du $\mathrm{XIX}^{\mathrm{e}}$ siècle, la forme habituelle de validation de prérogatives. Il sera alors fortement marqué par les nouveaux enjeux économiques et sociaux et le développement du prêt à intérêt. On en vint, sur la base de l'équivalence canoë/couvertures à faire " circuler » des canoës hors d'usage, voire fictifs, quand ce ne sont pas des parts de canoës : ce sont ces formes de la circulation des biens qui ont, dans les années 1920 , bloqué le potlatch lekwiltoq.

Parler du potlatch chez les Lekwiltoq (Lex̌wildax̌w), la tribu la plus méridionale des Kwagul (Kwakiutl) ${ }^{1}$, c'est traiter de l'institution qui a été décrite dans les années 1880 par Boas (Mauzé $1986: 31-40$ ) et analysée par ses nombreux commentateurs. C'est aussi revenir de manière plus détaillée sur une question ethnographique qui a été brièvement évoquée par P. Drucker et R. Heizer (1967) : il s'agit de l'utilisation systématique par les Lekwiltoq du canoë non seulement comme bien de prestige dans les distributions de type potlatch mais aussi comme référent symbolique. Au sein des autres groupes kwagul, au cours du xIx ${ }^{\mathrm{e}}$ siècle, le canoë est présent dans les potlatch, mais pas de façon générale et, vers 1900 , il y avait pratiquement disparu. Corrélativement au rôle qu'ils assignent au canoë, on observe que les Lekwiltoq, contrairement aux autres Kwagul méridionaux, ne faisaient pas usage des Cuivres, ou n'en firent usage que très tardivement, à partir des années 1910 seulement, et ce de manière exceptionnelle (ibid. $: 62)^{2}$. Nous aborderons ici la question du particularisme lekwiltoq à partir de cette variante locale des modalités du potlatch kwagul, et nous la situerons dans le contexte historique propre aux Lekwiltoq, analysé dans un récent travail (Mauzé 1985, II). 
Si la littérature ethnologique consacrée aux sociétés de la côte nord-ouest du Pacifique ménage une place de choix aux Kwagul, elle parle en réalité presque exclusivement des Kwagul de Fort Rupert, ou « vrais Kwagul », groupe auquel Boas et son informateur George Hunt ont particulièrement consacré leurs enquêtes et leurs travaux. Les Lekwiltoq avaient pourtant très tôt attiré l'attention. Dès les années 1820, il en est question dans les relations et les rapports des premiers observateurs blancs, traitants de la Compagnie de la baie d'Hudson ${ }^{3}$, puis voyageurs, explorateurs, missionnaires, agents administratifs de la Nouvelle-Calédonie qui allait devenir la Colombie britannique. Les Lekwiltoq se signalent aux premiers Européens par leur goût de la guerre : ils sèment la terreur, notamment parmi les populations salish du sud, capturent des esclaves en grand nombre et se lancent dans de lointaines expéditions de conquête (Mauzé 1985 : 368-377). Ainsi, dans la première moitié du XIX ${ }^{e}$ siècle, les Lekwiltoq entreprirent-ils d'occuper une partie du territoire des Salish Comox ${ }^{4}$ (Taylor \& Duff 1956 ; Mauzé 1985 : 342-367). Cette image que le monde blanc s'est forgée des Lekwiltoq est liée au fait que les premiers observateurs suivaient avec beaucoup d'intérêt le développement des guerres indiennes qui constituaient un obstacle non négligeable aux activités commerciales. Les premières observations dont nous disposons sur les guerres intertribales soulignent, à l'intérieur du monde des Kwagul méridionaux, une spécificité lekwiltoq sur laquelle les rapports administratifs des agents des Affaires indiennes de la fin du $\mathrm{XIX}^{\mathrm{e}}$ siècle continuent d'insister, alors que les guerres ont cessé depuis deux décennies.

Ce particularisme lekwiltoq se manifeste d'abord dans le caractère bien individualisé du liqwala parmi les dialectes du kwakwala ${ }^{5}$ et par un relatif isolement géographique au sein du territoire kwagul. Cet isolement s'explique par le fait qu'en entreprenant de conquérir et de coloniser les terres salish - ce mouvement de conquête s'étant accéléré après les premiers contacts avec les Blancs - les Lekwiltoq se sont trouvés à l'écart des grandes routes de traite durant la période du commerce maritime (1780-1830) et que, par la suite, ils se tiendront à l'écart des postes de la Compagnie de la baie d'Hudson établis le long de la côte continentale. Vivant dans des zones pauvres en animaux à fourrure, les Lekwiltoq n'ont jamais porté un grand intérêt au commerce de traite ; en revanche ils ont pratiqué le plus tard possible la chasse aux captifs de guerre qu'ils échangeaient contre des produits européens (Mauzé 1985 : 357). Nous avons montré que la société lekwiltoq que nous connaissons s'est constituée au moment des guerres salish, conduites dans le but d'obtenir des esclaves et de conquérir de nouveaux territoires. A partir d'un noyau initial de deux soustribus (les Wiwakai et les Wiwakam), les Lekwiltoq fusionnent avec un autre groupe d'origine kwagul, les Kwexa (Boas $1897: 332$; Curtis $1915: 108$ ) et absorbent, en les faisant passer sous leur domination, au moins un autre groupe d'origine salish, les Komenox, et même deux si l'on compte les Xaxamatsis (Walatsama) comme un groupe autonome ${ }^{6}$. A partir de 1820-1830, l'ensemble lekwiltoq regroupe cinq sous-tribus qui sont solidaires dans la 
guerre $^{7}$, solidarité sans cesse réaffirmée par des alliances matrimoniales internes à la tribu, mais associant des partenaires appartenant à des numaym de sous-tribus différentes. Dans ce contexte d'isolement, de croissance démographique et de développement des sous-tribus, les Lekwiltoq ont constitué une entité cérémonielle qui est demeurée repliée sur elle-même jusqu'à une époque relativement récente. Ils ont élaboré un système propre de hiérarchie de soustribus (et de numaym à l'intérieur de chacune d'elles) (Mauzé 1985 : 423-433) et des modalités particulières de financement du potlatch, dont la mise en forme la plus ancienne que nous connaissions concerne les années 18701880 , période où apparaissent les premiers grands potlatch intertribaux qui réunissent plusieurs tribus kwagul. La taille de la tribu lekwiltoq et sa division en sous-tribus lui permirent de conserver son autonomie cérémonielle dans la phase coloniale d'intensification des relations fondées sur le potlatch (Codere 1950).

Un aspect des modalités de mise en œuvre du potlatch illustre bien la place particulière que les Lekwiltoq occupaient au sein de l'ensemble kwagul : l'utilisation systématique des canoës en tant que biens de prestige distribués aux invités alors que les autres groupes kwagul utilisaient des Cuivres, notamment dans les potlatch dits de rivalité et les cérémonies de mariage à l'occasion du remboursement de la compensation matrimoniale au gendre par le beau-père (Boas 1895, 1921, 1966). On notera que cette absence des Cuivres est également attestée chez les Nootka (West Coast People) et les Salish côtiers (Drucker 1955 : 376, 431 ; Stott 1966 : 1 ; Widerspach-Thor $1981: 157)^{8}$. Pour ce qui concerne les Lekwiltoq, cette apparente anomalie se trouve justifiée par une légende qui raconte comment Wakai, l'ancêtre des Lekwiltoq, s'est fait ravir un coffre contenant des Cuivres que lui destinait son beau-père, le voleur étant un jeune homme de la tribu Nimpkish, investi de pouvoirs surnaturels, événement censé s'être produit au temps où les Lekwiltoq étaient établis dans un village à l'embouchure de la rivière Nimpkish (Boas 1895 : 145 ; Boas \& Hunt 1905 : 160-162 ; Mauzé 1985 : 318). Cette légende d'origine lekwiltoq et nimpkish explique l'absence de l'utilisation des Cuivres dans les potlatch de leur tribu et ratifie en creux l'importance que les autres tribus leur donnaient. Lorsque l'on sait que chez les Kwagul les Cuivres sont le symbole par excellence de la richesse ${ }^{9}$, ou que, par exemple, chez leurs voisins salish qui n'ont pas de Cuivres, c'est par le masque swaihwé qu'on peut acquérir la richesse (LéviStrauss 1979 : 144), on est évidemment tenté d'en déduire que, chez les Lekwiltoq, le canoë occupait la place sémantique et symbolique du Cuivre chez les Kwagul méridionaux ou du masque swaihwé chez les Salish. Cependant, au delà de ce que nous dit la légende, il est malaisé de fonder une hypothèse aussi générale et de nature fonctionnelle. Quitte à passer d'une hypothèse forte à une hypothèse faible, on peut tenter de solliciter l'ethnographie pour l'étayer.

Les « cérémonies à canoës » kwagul en général et lekwiltoq en particulier occupaient une place remarquable dans les mariages, qu'il s'agisse des modalités selon lesquelles les émissaires d'un homme à marier demandent la main de 
la jeune fille ou du transfert de la jeune épousée du village de son père à celui de son mari (Boas 1897 : 364 ; 1921 : 1051, 1065 ; 1966 : 54, 68). Selon certaines traditions locales, les membres de l'équipage du futur époux se mettent à chanter des chants de guerre à l'approche du village de la jeune épousée, signifiant ainsi qu'ils viennent capturer la mariée par la force (Holm \& Quimby $1980: 77)$; ou encore ce sont les hommes du village de la jeune fille qui, le jour du mariage, vêtus comme des guerriers, à bord d'un canoë de guerre, rassemblent tous les gens dans l'attente des « étrangers » (Curtis 1915: 125-126). Ce symbolisme guerrier du mariage contenu dans le terme winanem (« obtenir [une femme] dans la guerre ») est figuré par un simulacre de combat entre les groupes partenaires (Boas 1966 : 68). Chez les Lekwiltoq apparaîtrait ainsi une survalorisation du canoë dans ce contexte des rituels du mariage, au moins dans les séquences qui empruntent actes et paroles à la pratique de la guerre ; et comme le canoë est par excellence le canoë de guerre, on peut dire que les Lekwiltoq posent, à travers lui, l'équivalence entre mariage et guerre. Après avoir été de redoutables guerriers, ils n'ont jamais cessé d'exalter le souvenir de leurs actions militaires dont le canoë était le symbole. C'est ainsi que dans les potlatch, les canoës, les canoës de guerre en particulier, en seraient venus à être considérés comme les objets de prestige. Leur possession était le privilège des chefs et des personnages de haut rang : les gens du commun n'en possédaient pas (Boas 1895 : 87) ; parmi leurs prérogatives, les chefs comptaient des droits d'utilisation de noms ou de séries de noms pour les dénommer (Sapir 1979 : 115 ; Boas 1921 : 795-801 ; Drucker 1951 : 83, 84). Notons enfin que le canoë occupait une place importante dans les potlatch chez les Kwagul méridionaux au début du $\mathrm{XIX}^{\mathrm{e}}$ siècle : c'était un bien qui circulait d'un chef à l'autre (Codere $1950: 94$ ) et jouait un grand rôle dans les potlatch matrimoniaux, notamment dans les remboursements de la dot par la famille de la fille à celle du gendre (Goldman 1975 : 77) ; une phase de la cérémonie de remboursement était dite "couler le canoë » (Boas 1897: 422, 423, 518, 520) ${ }^{10}$. Pour le moment, il semble que c'est à partir du rôle réel joué par le canoë dans la guerre et de la représentation du mariage comme équivalent symbolique de celle-ci - mariage et guerre ayant à voir avec la « rivalité »- que doit s'orienter l'investigation, plutôt que vers la recherche d'équivalences fondées sur la mythologie : entre canoë et Cuivre d'une part, entre canoë et masque swaihwé (xwéxwé) d'autre part, même si, a priori, on ne saurait renoncer à cette possibilité.

A la lumière des données dont nous disposons, nous pouvons décrire et analyser les traits caractéristiques de ce potlatch à canoës chez les Lekwiltoq. Ceux-ci les distribuaient dans tous les contextes cérémoniels à potlatch : dation d'un nom à un enfant, initiation dans une société secrète, mariage, funérailles, etc. De la même façon qu'un Cuivre, un canoë est identifié par un nom $^{11}$ qui se perpétue lors de son changement de propriétaire. Un canoë peut être donné, brisé, brûlé, mais aussi, dans le contexte du prêt à intérêt qui s'est développé à la fin $\mathrm{du} \mathrm{XIX}^{\mathrm{e}}$ siècle - opération désormais préliminaire au 
potlatch -, vendu, prêté ou emprunté. Le peu que l'on sait des modalités anciennes de cession par don, vente ou prêt indique que ces transactions devaient être accomplies dans le respect de règles précises, qu'il nous est malheureusement impossible de reconstituer aujourd'hui, tant les observations à cet égard ont été fragmentaires. Retenons que chez les Lekwiltoq les canoës intervenaient de deux manières dans les potlatch : comme biens de prestige susceptibles d'être donnés et comme objets-étalon dans les transactions du type achat-vente ou emprunt-prêt, dans lesquelles étaient en permanence engagés les chefs et personnages de haut rang et tous les membres des numaym des soustribus solidaires de leur chef, ce second aspect du rôle des canoës ayant pris une importance considérable à la fin du $\mathrm{XIX}^{\mathrm{e}}$ siècle, époque de l'individualisation du potlatch.

Cet objet de prestige que nous appelons ici « canoë », qu'était-il ? La question étonnera et pourtant elle se pose, ne serait-ce que dans la mesure où nous ne savons pas si ces « canoës » étaient de véritables embarcations de pêche, de guerre ou de navigation à longue distance utilisées dans les potlatch alors qu'elles étaient encore en état de fonctionnement ou après avoir été désaffectées, ou bien s'il s'agissait en totalité ou en partie d'engins ayant pour seule destination l'utilisation cérémonielle. Au moins sait-on que parmi ceux-ci il y avait de vrais canoës de guerre (Drucker \& Heizer 1967 : 62 ; McKennie 1972 : 36). Ils étaient fabriqués par les Lekwiltoq eux-mêmes qui rappelaient ainsi qu'ils étaient d'excellents constructeurs (Drucker, s.d.). Jusque vers les années 1870, dans le village de Cape Mudge (village wiwakai), chaque homme fabriquait en moyenne deux canoës par été ; les achats pratiqués auprès des Cowichan de la côte orientale de l'île de Vancouver ou auprès des Salish de la région du Puget Sound (ibid.) ne datent peut-être que des années 1860-1870, marquées par la fin des guerres inter-tribales. Mais très rapidement, au tournant du siècle ou même avant, ces canoës, qui avaient pu être utilisés comme tels, n'intervenaient plus que comme unités de compte ou valeurs de référence dans les diverses transactions du potlatch. Les canoës " réels " avaient complètement disparu ou, au mieux, n'existaient plus qu'à l'état de vieilles carcasses pourrissantes, abandonnées sur la plage (Drucker \& Heizer 1967). Cependant, ils continuaient d'avoir une « existence de potlatch » du fait de leur identification par un nom et de la circulation à leur place de leur équivalent-couvertures.

On sait qu'à partir des années 1850 les marchandises européennes avaient commencé de supplanter les objets fabriqués par les Indiens ; parmi les objets importés, il en est un qui jouera un rôle considérable dans l'économie indienne en général et dans celle du potlatch en particulier : c'est la couverture de la Compagnie de la baie d'Hudson. Les biens de prestige seront donc évalués d'abord en couvertures, puis les couvertures se substitueront progressivement aux biens traditionnels : ainsi les canoës pourront-ils continuer à " circuler " même s'ils n'existent plus ; mieux : on peut penser que ce système d'équivalence a permis d'augmenter le nombre de " canoës » sans avoir à en fabriquer. Un de nos informateurs rend compte de cette situation dans les termes suivants, 
à propos d'un canoë appelé White Bottom : "Il était question de White Bottom, mais finalement White Bottom n'existait pas. Ils [les Lekwiltoq] étaient pris dans ce système qui était considéré comme normal à l'époque. " Un autre informateur confirme cette remarque : «Il n'y avait plus de canoës, mais cependant on parlait d'eux. "

Contrairement à ce qu'ont prétendu Drucker et Heizer (1967: 62), une raréfaction temporaire de canoës " réels " n'entraînait pas une augmentation de leur valeur unitaire : la possibilité de créer des canoës fictifs permettait de maintenir constants leur nombre, donc leur valeur qui, dans les années 18801920, était calculée en couvertures d'un dollar chacune. Dans les potlatch, la valeur-couvertures d'un canoë était indépendante des caractéristiques de celuici : un canoë « réel » neuf pouvait fort bien valoir le même nombre de couvertures qu'un canoë hors d'usage ou fictif. En 1876, un canoë de guerre neuf brûlé au cours d'un potlatch valait 175 dollars (McKechnie 1972 : 36) ; dans les années 1900-1910, selon nos informateurs, un canoë de taille moyenne (25 pieds) en valait 150 tandis qu'un petit en valait 100. Vers 1915-1920, un homme paie le montant de la compensation matrimoniale au frère de sa femme avec 35 canoës d'une valeur de 100 dollars chacun (100 couvertures), auxquels sont ajoutées 1000 couvertures (Mauzé 1985 : 436-437) ; nous demeurons dans les ordres de grandeur évoqués ci-dessus ${ }^{12}$. Ici apparaît une différence fondamentale entre la circulation des canoës chez les Lekwiltoq et celles des Cuivres chez les autres Kwagul méridionaux : alors que la valeur d'un Cuivre augmente chaque fois qu'il change de main, celle du canoë demeure stable. Dans le potlatch à canoë (sa'akya) (" don de canoë "; Boas $1966: 81$ ) ne pouvait varier que le nombre de canoës à distribuer en fonction de l'enjeu du potlatch. Une fois admis que par " canoë » il faut entendre "quelque chose " qui a un nom, une valeur d'échange, qui est susceptible d'être distribué quoi qu'il en soit de sa matérialité, le don de canoës ne diffère plus fondamentalement du don de couvertures.

Les faits se compliquent lorsqu'on envisage les modalités de prêt et d'emprunt qui constituent à la fin du XIx ${ }^{\mathrm{e}}$ siècle la phase préliminaire de la distribution. En effet, pour pouvoir donner des canoës, il faut s'en procurer, ce qui ne saurait se faire que par achat ou emprunt. Compte tenu que le système de prêt fonctionnait pour l'essentiel en vase clos, que la population subissait alors une forte baisse démographique - au début des années 1880 on dénombre 400 Lekwiltoq, en 1910, 220, et en 1920, 145 (Mauzé 1985 : 552) et que le nombre des positions hiérarchiques pour les onze numaym des quatre sous-tribus était de l'ordre de 180 dans les années 1910 (ibid. : 426), il est aisé de comprendre que les partenaires se trouvèrent dans la double position de débiteur et de créditeur. Pour acquérir un canoë entier ou une "part " de canoë, il suffisait de verser un acompte sur le total, généralement évalués l'un et l'autre en couvertures. Le seul paiement d'un acompte donnait le droit d'aliéner le canoë - ou la "part »- partiellement acheté. Une même " chose » ainsi appropriée partiellement pouvait être vendue, de telle sorte que 
ventes et achats en série se succédaient pour un même objet : un même canoë pouvait figurer à plusieurs reprises dans les livres de compte (potlatch books), comme celui dont nous avons pris connaissance à Cape Mudge ${ }^{13}$, puisque divers acquéreurs en revendiquaient en même temps et légitimement la propriété. Un canoë pouvait passer entre les mains de quatre ou cinq propriétaires sans avoir été payé complètement au propriétaire initial. L'acompte s'élevait à 15 couvertures pour un petit canoë, à 40,60 , voire 60 pour un grand. Mais son montant ne dépendait pas seulement de la taille du canoë, il était aussi calculé en fonction du statut social du nouveau demandeur : un acheteur de rang élevé versait davantage de couvertures qu'un acquéreur de moindre statut. Le crédit ouvert par le vendeur fonctionnait comme dans les autres tribus des Kwagul méridionaux : il était rémunéré par un intérêt qui variait selon la durée du prêt. Généralement le nouvel acquéreur devait rendre au double la valeur du canoë. Tout était mis en œuvre pour « faire travailler» les canoës, comme l'indiquent les Lekwiltoq aujourd'hui encore.

Nos informateurs comparent la manière dont s'effectuaient ces transactions au fonctionnement d'une sorte de «Wall Street» à l'échelle de la tribu. La perspective de l'organisation d'un potlatch était l'occasion d'une mise au net de l'état des créances et des dettes. Pour l'invitant, il s'agissait de tout mettre en œuvre pour « faire rentrer les canoës », c'est-à-dire obliger ses débiteurs à rembourser leurs dettes. A Cape Mudge, par exemple, quelques jours avant la date prévue pour la manifestation, toutes les personnes concernées se réunissaient en plein air. On s'asseyait en cercle, chacun ayant un ou plusieurs ballots de dix ou quinze couvertures qui passaient de main en main au rythme de l'apurement des comptes. Un contrôleur (tally man) dessinait sur le sol un plan du village, les maisons étant représentées par des rectangles alignés, comme celles-ci, face à la mer. Chaque rectangle était divisé en autant de fractions que la maison comprenait de familles nucléaires, une par « coin » en général. A l'intérieur des subdivisions figurait le nombre initial de canoës détenus par le chef de famille, chaque canoë étant représenté graphiquement en coupe par un dessin en forme de « $U$ » évasé. A mesure que s'opéraient les transactions, les canoës, identifiés avec autant de précision que les maisons, se déplaçaient de « coin » en " coin " et de maison en maison au moyen de dessins effacés ici, reproduits là. A la fin, le dessin d'ensemble rendait compte de la nouvelle répartition des canoës. Lorsqu'on commença à disposer de tally men lettrés, on consigna ces transactions dans des registres ou potlatch books.

Il va sans dire qu'expliqué de cette manière le fonctionnement du système apparaît relativement simple. Cependant, les relations entre les partenaires se trouvaient compliquées du fait même que la notion de canoë unitaire devenait problématique, en raison non seulement de la circulation généralisée de canoës fíctifs et de la possibilité d'en " créer », mais encore de la double démultiplication de ce qui circulait. D'une part nous passons d'une circulation de canoës à une circulation de parts de canoës, d'autre part nous avons un nombre de propriétaires de canoës ou de parts de canoës nécessairement très supérieur au 
nombre de partenaires réels en vertu du principe selon lequel l'acompte versé sur un achat suffit à engager une vente.

Un système fondé sur cette fuite en avant en quoi consistait la généralisation du crédit n'était évidemment pas viable. La comptabilité des cessions de canoës ou de parts de canoës qui n'étaient pas entièrement payés et passaient entre les mains de plusieurs acquéreurs était très complexe, à la limite impossible à gérer par la seule tenue des livres. On en vint, à l'instar des Tswadenox̌w de Kingcome Inlet (Drucker, s.d.), à faire circuler des « papiers » qui étaient des engagements à terme : après les canoës fictifs, mais matérialisés par des couvertures ou par leur valeur en dollars, on en arriva à une circulation de biens entièrement fictive. Même dans ce système extravagant, il fallait compter avec les « mauvais payeurs » et les « spéculateurs », c'est-à-dire avec ceux qui, après avoir maximisé leurs emprunts, se dérobaient à l'obligation de rembourser. On raconte qu'une femme mit en péril en « jouant à la bourse » (sic), en l'occurrence en achetant à crédit, en vue de payer la compensation matrimoniale due par son fils à son futur beau-père, un nombre de canoës tel qu'elle se trouva dans l'incapacité d'honorer ses dettes. Vers 1920, ce système était bloqué. Un chef de Cape Mudge tenta de calmer le jeu en achetant un Cuivre dans l'intention de faire cesser les conflits qui éclataient à l'occasion de chaque potlatch (Drucker \& Heizer : 1967 : 62) et peut-être aussi de faire redémarrer le système du potlatch sur des bases nouvelles, mais cette initiative ne fut jamais renouvelée (ibid.).

La confiscation des objets cérémoniels en 1922 (LaViolette 1973 : 44 sq. ; Mauzé 1983 ; 1985 : 295-304), le contexte général de répression dans lequel elle s'inscrivait, l'entrée des communautés indiennes dans la période d'organisation clandestine des cérémonies firent disparaître une " économie » de potlatch qui était devenue incontrôlable. Dans une large mesure, cette disparition fut le résultat de l'action des Indiens eux-mêmes : de ceux qui, nouveaux entrepreneurs soutenus par les missionnaires, n'envisageaient plus leur survie socio-économique que dans la concurrence avec le monde blanc : l'esprit de compétition du potlatch pouvait encore être mobilisé, mais à d'autres fins. C'est l'exemple que les Lekwiltoq nous offre depuis soixante ans.

CNRS, Paris

Laboratoire d'anthropologie sociale

NOTES

1. Les Kwagul méridionaux sont répartis en une vingtaine de tribus, elles-mêmes divisées en unités sociales plus petites auxquelles Boas a donné successivement plusieurs noms : " gens », en référence à une orientation patrilinéaire de la filiation ; "clan », pour marquer la présence de traits matrilinéaires ; numaym, enfin, utilisant le terme indigène qui signifie « gens de la même sorte ». Pour rendre compte de la nature du numaym, C. LÉvi-Strauss (1979) utilise le concept de " maison". 
2. Au cours d'un potlatch auquel ils avaient été conviés, et sur lequel nous sommes mal renseignés, deux ou trois chefs lekwiltoq reçurent en cadeau des « faux » Cuivres sans valeur ou encore, pour employer l'expression de Hunt, des "smooth faced coppers ». Ces faux Cuivres faisaient partie d'un lot de treize pièces d'une valeur marchande unitaire de 100 dollars qui, avec 2700 couvertures, représentaient la valeur d'un Cuivre appelé "Long Between Killerwhale » (HưNT, s.d. : 46, 73). Ces faux Cuivres n'avaient en vérité ni fonction symbolique ni fonction rituelle (WIDERSPACHTHOR 1981).

3. On peut consulter à cet égard les journaux de Fort Langley tenus entre 1827 et 1830 , qui constituent les premières sources d'information sur les Lekwiltoq.

4. Les linguistes distinguent deux dialectes du comox : le comox continental parlé par les Homalco, les Klahoose et les Sliammon, et le comox insulaire dont les locuteurs sont les Comox proprement dits (HAGÈGE 1981 ; KeNNEDY \& BouCHARD 1983).

5. On distingue généralement deux sous-dialectes du kwakwala : le sous-dialecte septentrional parlé par les groupes habitant la côte nord-ouest de l'île de Vancouver et les îles littorales de la côte continentale au nord de Blunden Harbour, et le sous-dialecte méridional dont les locuteurs vivent entre Port-Hardy et Campbell River sur la côte est de l'île de Vancouver, au sud de Blunden Harbour sur la côte continentale, et dans les îles du détroit de Johnstone, au sud de l'île Malcolm (BoAs 1947, 1966 ; Wuson, s.d.).

6. L'origine ethnique des Xaxamatsis ou Walatsama fait problème : il est difficile de savoir, en l'état des informations disponibles, si ce groupe est d'origine comox ou kwagul (MAUzÉ 1985 : 403-406). La sous-tribu komenox s'est éteinte vers 1860 ; les survivants ont fusionné avec les Wiwakam.

7. Bien qu'il soit généralement admis que chaque groupe local est indépendant des groupes voisins pour ce qui est de la guerre (DrUCKER $1983: 88$ ), il semble que les sous-tribus lekwiltoq menaient ensemble leurs expéditions et faisaient bloc face aux raids de représailles lancés par les tribus salish, parfois associées pour l'occasion. Les expéditions lekwiltoq réunissaient entre 200 et 400 guerriers à la fin des années 1820 (Mauzé $1985: 371,372$ ).

8. Barnett (1955: 266) note que des Cuivres de peu de valeur ont circulé chez les Comox ; leur valeur restait stable : cent à deux cents couvertures ; ils n'étaient jamais brisés.

9. Les mythes kwagul relatent comment certains individus reçurent le Cuivre de Dzonoqwa, l'ogresse, et de Qomoqwa, chef du monde sous-marin et maître de toutes les richesses ; Qomoqwa porte aussi le nom de Tlaqwagila « Faiseur de Cuivre ».

10. Le « canoë » en question est figuré par un carré délimité sur le sol au moyen de pièces de bois peintes incrustées de dents de loutre (gisexstala) rappelant les couvercles du coffre à l'intérieur duquel sont déposés les biens donnés au gendre par le beau-père. Lorsque tous les biens ont été rassemblés, le « canoë » est fendu à un coin du carré à l'aide d'une hache. Les biens peuvent alors être distribués aux membres du numaym du gendre.

11. Drucker (Field Notes) a recueilli dans les années 1950 quelques noms de canoës ; ainsi : dasd'ala « Laugh at » (would be buyer) ; wilwasu « Can't be bought »; ginanamas « Too Young " (to be bought). Les notes de terrain de Drucker ont été utilisées avec la permission des Archives de la Smithsonian Institution, que je remercie.

12. Cette stabilité des prix est confirmée par HuNT dans son manuscrit : History of 21 Coppers.

13. Le Potlatch Book de Cape Mudge que nous avons pu consulter est un livre de compte dans lequel sont consignées les transactions de canoës (ainsi que de vaisselle, de châles, etc., pour les femmes) à propos de potlatch qui eurent lieu entre 1911 et 1916. Ce registre était à l'origine un journal de bord faisant partie du butin que les Wiwakai se partagèrent à la suite du naufrage du Cottage City, navire marchand battant pavillon américain qui s'était échoué en janvier 1911 dans le Passage de la Découverte, en face de Cape Mudge. 


\section{B I B L IO G R A P H I E}

BARnetT, H. G.

1955 The Coast Salish Indians of British Columbia. Eugene, University of Oregon Press.

BOAs, F.

1895 Indianische Sagen von der Nord-Pacifischen Küste Amerikas. Berlin, A. Asher (trad. angl. non publ., Indian Language Project, Victoria, BC, 1977).

1897 «The Social Organization and the Secret Societies of the Kwakiutl Indians », Report of the United States National Museum for 1895. Washington DC, Smithsonian Institution.

1921 Ethnology of the Kwakiutl. Bureau of the American Ethnology, Thirty-fifth Annual Report, Part 1 \& 2. Washington DC., Smithsonian Institution.

1925 Contributions to the Ethnology of the Kwakiutl. New York, Columbia University Press («Columbia University Contributions to Anthropology »3).

1947 «Kwakiutl Grammar with a Glossary of the Suffixes ». Edited by Helene Boas Lamposky with the collaboration of Z. Harris. Transactions of the American Philosophical Society, n.s., 37, part $3: 203-377$.

1966 Kwakiutl Ethnography. Edited by H. Codere, Chicago \& London, University of Chicago Press (" Classics in Anthropology »).

BOAs, F. \& G. HUNT

1905 Kwakiutl Texts (Publications of the Jesup North Expedition, 3). New York, G. E. StechertLeiden, Brill (« Memoirs of the American Museum of Natural History »).

CODERE, $\mathrm{H}$.

1950 Fighting with Property. New York, J. J. Augustin (« Monographs of the American Ethnological Society »18).

Curtis, E.

1915 The North American Indians. The Kwakiutl, 10. Nordwood, Plimpton Press.

DRUCKer, P.

1951 The Northern and Central Nootkan Tribes. Washington DC, Smithsonian Institution (« Bulletin » 144).

1955 Indians of the Northwest Coast. New York, The Natural History Press.

1983 «Ecology and Political Organization on the Northwest Coast of America » in E. TookER, ed., The Development of Political Organization in Native North America. Washington, DC, American Ethnological Society (" Proceedings of the American Ethnological Society ").

s.d. Field Notes (1953). Archives of the Bureau of the American Ethnology, Washington DC, Smithsonian Institution, Add. Mss. 870 (Ms. 4516 [2] vol. 6).

DRUCKER, P. \& R. HEIZER

1967 To Make My Name Good. A Re-examination of the Southern Kwakiutl Potlatch. Berkeley, University of California Press.

DUFF, W.

s.d. Notes. Victoria, BC, British Columbia Provincial Archives.

GoldmaN, I.

1975 The Mouth of Heaven. An Introduction to Kwakiutl Religious Thought. London, John Wiley \& sons.

HAGÈGE, C.

1981 «Le Comox Lhaamen de Colombie britannique », Paris, Amerindia 2, n spéc.

Holm, B \& G. QUImBY

1980 Edward S. Curtis in the Land of the War Canoes. Seattle, University of Washington Press ("Thomas Burke Memorial Washington State Museum Monograph » 2). 
Hunt, G.

s.d. History of 21 Coppers from Alert Bay (1924 ?). (Boas Collection 372 ; roll 3). Philadelphia, American Philosophical Library.

KENNEDY, D. \& R. BOUCHARD

1983 Sliammon Life, Sliammon Lands. Vancouver, Talonbooks.

LAVIOLETTE, F.

1973 The Struggle for Survival. Toronto. University of Toronto Press.

LÉvi-Strauss, C.

1979 La Voie des masques. Édition augmentée. Paris, Plon.

MAUZE, $M$.

1983 « La loi anti-potlatch chez les Kwagul », Bulletin Amérique indienne, juil. : 3-5 ; août-sept. : 9-11.

1985 Enjeux et jeux du prestige. Des Kwagul méridionaux aux Lekwiltoq (côte nord-ouest du Pacifique). Thèse pour le doctorat de $3^{e}$ cycle. Paris, EHESS, 2 vol.

1986 « Boas, les Kwagul et le potlatch », L'Homme 100, XXVI (4) : 21-63.

McKeChNIE, R. A.

1972 Strong Medecine. Vancouver, J. J. Douglas.

SAPIR, E.

1979 "L'Organisation sociale des Indiens de la côte ouest ", in Anthropologie 2. Paris, Éd. de Minuit ("Le Sens commun ») : 107-132.

STotr, M.

1966 « The Southern Kwakiutl Copper. A Study based on the George Hunt Manuscript », B.A., Department of Anthropology, University of British Columbia.

TAXLOR, H. \& W. DUFF

1956 « A Post-Contact Southward Movement of the Kwakiutl 》, Research Studies 24 (1) : 56-66.

WALENS, $S$.

1981 Feasting with Cannibals. An Essay on Kwakiutl Cosmology. Princeton, Princeton University Press.

WIDERSPACH-THOR, $M$.

1981 " The Equation of Copper ", in Donald ABbort, ed., The World Is as Sharp as a Knife. Victoria, British Columbia Provincial Museum : 157-174.

Wrson, P.

s.d. The Kwakwala language, ms.

ARCHTVES

Hudson Bay Company Archives, Winnipeg. Fort Langley. Post Journal, 1827-1830, B 113/a. 


\begin{abstract}
A B S T R A C T
Marie Mauzé, Canoes in Lekwiltoq Potlach. - The Lekwiltoq, one of the major tribes of the southern Kwagul, give to the canoe a place in their potlach that is held by the Coppers among other groups. Although a myth seems to explain why Copper is missing in Lekwiltoq potlach, we cannot, on the basis of ethnographic studies, consider Copper and Canoes to be equivalent, despite ample evidence that the latter are important, for social and ceremonial reasons, in this warrior society. Canoe potlach, which has almost disappeared among the Kwagul, has been maintained among the Lekwiltoq ; it became the habitual way to validate prerogatives in the late 19th century, when it was strongly marked by new economic and social developments, notedly by recourse to interest-bearing loans. Through the equivalence between canoes and blankets, canoes (or even parts of them) that were no longer useable, or were even fictive, were put into "circulation"; and as a consequence, the Lekwiltoq potlach system was blocked in the 1920 s.
\end{abstract}

\title{
ZUSAMMENFASS UNG
}

Marie Mauzé, Das Kanu im lekwiltoq Potlatch. - Die Lekwiltoqs, einer der Hauptstämme der Südkwaguls, sprechen dem Kanu in ihren Potlatch die gleiche Stelle zu, die das Kupfer in anderen Gruppen einnimmt. Scheint ein Mythus die Abwesenheit des Kupfers im lekwiltoq Potlatch zu rechtfertigen, erlaubt die Ethnographie jedoch nicht die Behauptung, dass das Kanu dem Kupfer entspricht, auch wenn die Wichtigkeit des Kanus im zeremoniellen und sozialen Leben dieser kriegerischen Gesellschaft wohl bestätigt ist. Der Potlatch des Kanus, der bei den anderen Kwaguls fast verschwunden ist, bleibt im Gegenteil bei den Lekwiltoqs erhalten, um am Ende des 19. Jahrhunderts die gewöhnliche Form der Gültigkeitserklärung der Vorrechte zu werden. Von da an wird er von den neuen wirtschaftlichen und sozialen Einsätzen und von der Verbreitung des verzinslichen Darlehens geprägt. Man kam dazu, aufgrund der Äquivalenz Kanu/Decken ausserbetriebliche oder fiktive Kanus " schwimmen " zu lassen, wenn es nicht Teile von Kanus sind : es sind Formen des Güterverkehrs die in den 20er Jahren den lekwiltoq Potlatch blockiert haben.

\section{R E S U M E N}

Marie MAUzÉ, La Canoa en el potlatch lekwiltoq. - Los Lekwiltoq, una de las principales tribus de los Kwagul meridionales, assignan a la canoa en sus potlatch el lugar que ocupan los Cobres en los otros grupos. Si un mito parece justificar la ausencia de Cobres en el potlatch lekwiltoq, la etnografía no permite considerar equivalentes la canoa y el cobre, incluso si esta bien probada la importancia de la canoa en la vida social y ceremonial de esta sociedad guerrera. Desaparecido practicamente entre los otros Kwagul, el potlatch de canoa por el contrario se mantiene entre los Lekwiltoq hasta llegar a ser, a finales del siglo xIx, la forma habitual de valorar las prerrogativas. Se vera por tanto afectado por los nuevos intereses económicos y sociales y el desarrollo del préstamo con interés. Se llega, en base a la equivalencia canoa/manta, a hacer "circular " canoas fuera de uso, incluso ficticias, cuando no son partes de ellas. Estas formas de circulación de bienes han bloqueado el potlatch lekwiltoq en los años 1920. 